\title{
Col du lac Blanc : un site pour l'observation et la modélisation de la neige en haute montagne
}

\author{
Vincent Vionnet ${ }^{1,2}$, Florence Naaim-Bouvet ${ }^{3}$, Yannick Deliot ${ }^{1}$, \\ Hervé Bellot ${ }^{3}$, Fatima Karbou', Mohamed Naaim ${ }^{3}$, Gilbert Guyomarc'h', \\ Yves Durand ${ }^{1}$ \\ 1. Centre d'études de la neige, Centre national de recherches météorologiques, \\ Météo-France / CNRS, Saint-Martin-d'Hères \\ 2. Centre for Hydrology, University of Saskatchewan, Saskatoon, Canada \\ 3. Unité de recherche érosion torrentielle neige et avalanche, Université Grenoble \\ Alpes / Irstea, Saint-Martin-d'Hères \\ fatima.karbou@meteo.fr
}

\section{Résumé}

Depuis 1990, Météo-France et l'Irstea gèrent le site expérimental du col du lac Blanc situé à $2700 \mathrm{~m}$ dans le massif des Grandes Rousses dans les Alpes françaises. Ce site a plusieurs atouts qui le rendent unique dans le paysage des sites d'observations en France et en Europe. Par sa localisation, le site est dominé par des vents orientés nord-sud et il est sans surprise particulièrement bien adapté pour l'observation et la modélisation des effets du transport de neige par le vent, grâce à une panoplie d'instruments de mesures. Dans cet article, nous ferons une présentation générale du site observatoire en détaillant les grandeurs de neige et atmosphériques mesurées qui lui ont valu plusieurs labellisations nationales et internationales. Un aperçu des recherches menées à partir des données ou des équipements du site expérimental est également donné. L'accent est mis sur les recherches visant une modélisation réaliste de l'effet du transport de neige par le vent dans différents modèles et d'études pilotes pour l'exploitation des mesures de télédétection en haute montagne. e transport de la neige par le vent est une composante importante de l'interaction entre la cryosphère et l'atmosphère. Il se manifeste dans les régions couvertes de neige de manière saisonnière ou permanente. En zone alpine, il a en premier lieu des conséquences sur la formation des avalanches (Schweizer et al., 2003). Le transport entraîne en effet une redistribution hétérogène de la neige, fortement influencée par la topographie locale (par exemple, Schirmer et al., 2011). La neige est érodée dans les zones exposées au vent, à proximité des crêtes par exemple, et tend à se déposer dans les dépressions à l'abri du vent. Au cours de leur transport, les particules de neige se fragmentent (Comola et al., 2017) et se déposent sous forme de couches présentant une grande cohésion. Lorsqu'elles reposent sur une couche de faible cohésion, le passage d'un skieur peut alors suffire à provoquer l'effondrement de la sous-couche fragile et la mise en mouvement de l'ensemble des couches de neige déposée : $65 \%$ des accidents d'avalanche s'observent après un épisode de transport de neige par le vent (chiffre de l'Association nationale d'étude de la neige et des avalanches). Outre le danger d'avalanche, la redistribution de la neige sous l'effet du vent a des conséquences en termes de viabilité hivernale (formation de congères) et en termes d'hydrologie (Winstral et al., 2013). En effet, elle s'accompagne de la sublimation des particules de neige transportée entraînant une perte d'eau (Schmidt, 1982 ; Groot Zwaaftink et al., 2011).
De nombreux modèles de transport de la neige par le vent ont été développés pour simuler les processus mis en œuvre lors des épisodes de transport et la redistribution de la neige en résultant (par exemple, Naaim et al., 1998 ; Durand et al., 2005 ; Liston et al., 2007 ; Lehning et al., 2008 ; Mac Donald et al., 2010 ; Schneiderbauer et Prokop, 2011). Cependant, le transport éolien de neige est un processus encore mal pris en compte dans ces modèles qui ne représentent que de manière simplifiée la physique complexe de ce phénomène (Askamit et Pomeroy, 2016). Pour améliorer les connaissances dans ce domaine, les études in situ sont indispensables et indissociables des recherches fondamentales en laboratoire. Elles permettent d'une part de mieux décrypter les processus physiques en jeu et d'autre part de tester les hypothèses et de valider les modélisations numériques. C'est pourquoi, depuis les années 1990, Météo-France et l'Irstea (Institut national de recherche en sciences et technologies pour l'environnement et l'agriculture) se sont associés pour développer le site expérimental du col du lac Blanc, un laboratoire d'altitude situé à $2700 \mathrm{~m}$ dans les Alpes françaises et particulièrement venté. À son lancement, ce site était plutôt conçu pour réaliser des campagnes de mesures ponctuelles. Par la suite, de plus en plus de mesures ont été réalisées, pour certaines assez rares comme les mesures de transport de neige par le vent. Sa reconnaissance est désormais internationale, notamment par ses récentes labellisations en tant qu'observatoire. Il est vrai que les 


\section{Abstract}

Col du Lac Blanc: a site for snow observing and modeling in high mountains

Since 1990, Météo-France and IRSTEA have been managing the experimental site of the Col du Lac Blanc located at $2700 \mathrm{~m}$ altitude in the Grandes Rousses massif in the French Alps. This site has several advantages that make it unique among other observation sites in France and in Europe. By its location, the site may be considered as a natural wind tunnel and not surprisingly was mainly dedicated to observation and modeling studies on wind-induced snow transport. In this article, we make a general presentation of the Col du Lac Blanc site facilities with description of relevant snow and atmospheric measurements. Then an overview of research studies made using data from this experimental site or using its facilities will be given. A focus will be made on modeling studies towards a realistic representation of wind-induced snow transport in different models and on some pilot studies to enhance the use of remote sensing date in mountains. mesures détaillées de transport de neige par le vent restent rares sur le plan international. Mais le vent n'est pas le seul atout du col du lac Blanc. Altitude élevée, facilité d'accès et logistique adaptée en font un site particulièrement attractif pour mener des recherches sur la neige en haute montagne. Cet article présente donc les caractéristiques techniques du col du lac Blanc et la base de données correspondante, ainsi que ses potentialités passées et futures à travers des exemples de recherches.

\section{Description générale du site et données de long terme}

Le col du lac Blanc est situé dans le massif des Grandes Rousses à $2720 \mathrm{~m}$

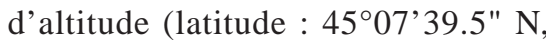
longitude : $06^{\circ} 06^{\prime} 41.599^{\prime \prime} \mathrm{E}$, figure 1a). Il est bordé à l'est par la chaîne principale des Grandes Rousses et à l'ouest par le dôme des Petites Rousses (figure 1b). Cette configuration topographique particulière assure une canalisation des vents au niveau du col suivant un axe nord-sud.

Le site expérimental du lac Blanc est composé de quatre stations de mesures indépendantes : Lac Blanc, Dôme, La Muzelle et Col (voir la figure 1c et la figure 2 pour la localisation des stations). Les trois premières stations sont gérées par Météo-France, la station Col est sous la responsabilité de l'Irstea. Les stations Lac Blanc, La Muzelle et Col sont situées à proximité immédiate du col, alors que la station Dôme est située au sommet du dôme des Petites Rousses. Cette dernière apporte des informations précieuses sur les conditions atmosphériques en dehors de la zone de canalisation de l'écoulement rencontrée à proximité du col. Le tableau 1 liste les mesures atmosphériques et de neige effectuées à l'ensemble des stations. L'influence de la topographie sur les vents au col du lac Blanc est illustrée par la rose des vents obtenue pour la station Lac Blanc pour la période 2010-2016 (figure 3).

\section{a) Localisation générale b) Massif des Grandes Rousses}

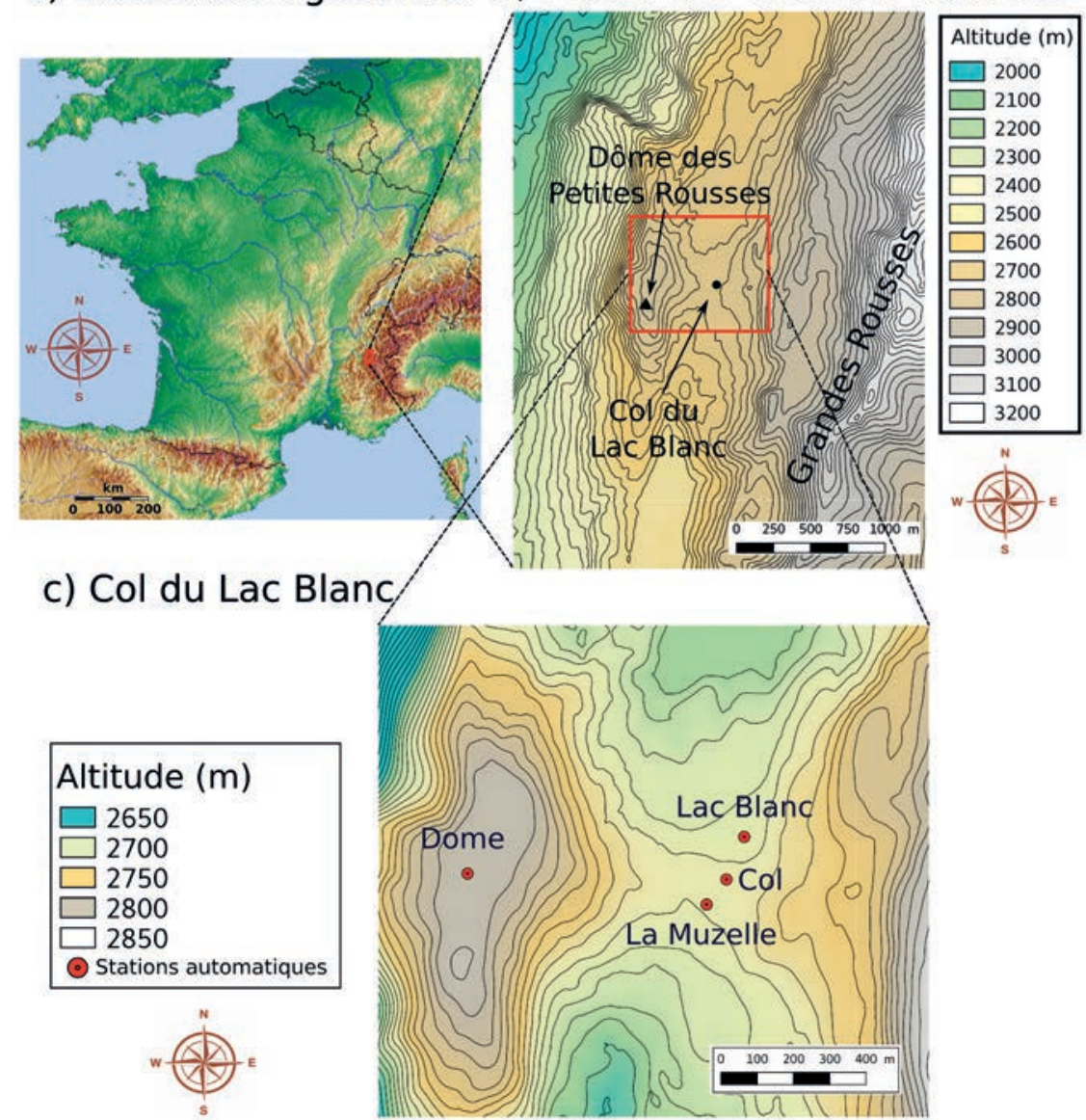

Figure 1. Localisation (a) et détail (b, c) de la topographie autour du col du lac Blanc. La carte (c) montre la localisation des quatre stations automatiques installées à proximité du site. 


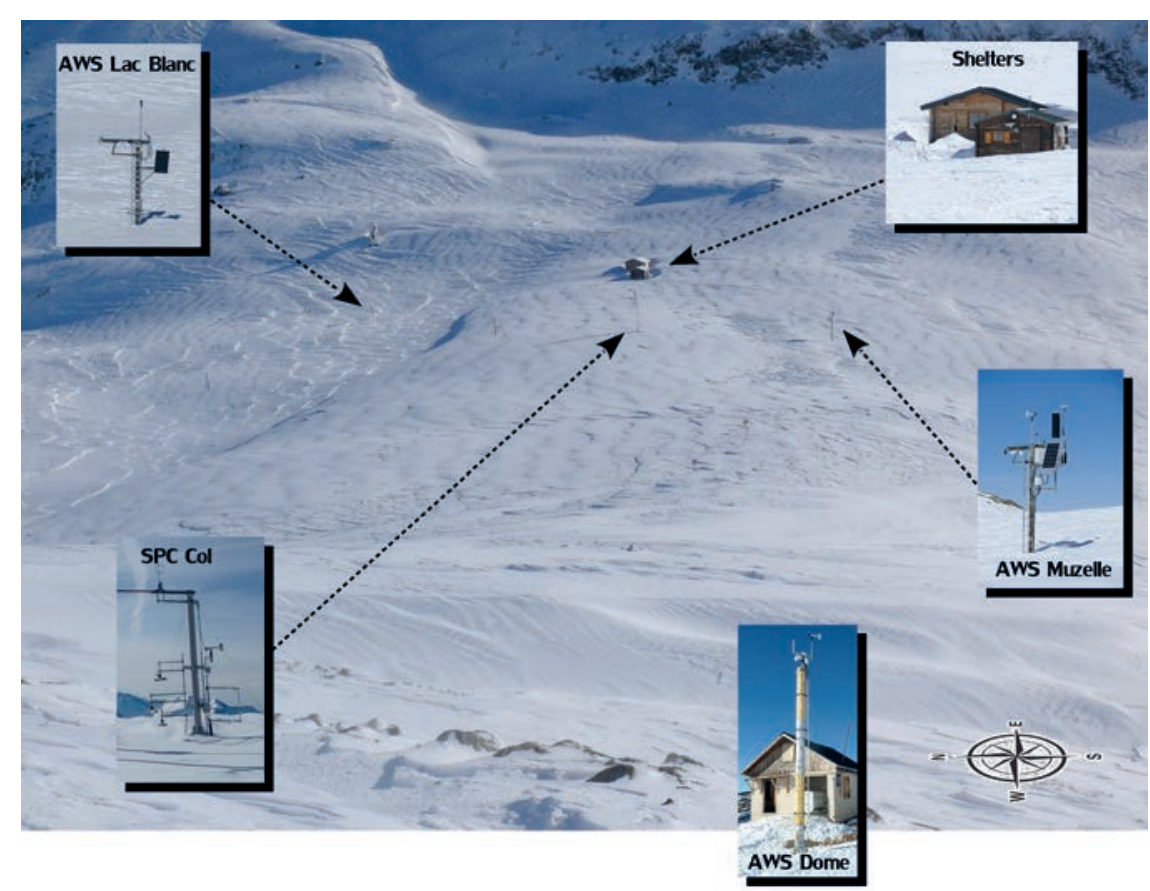

Figure 2. Illustration du dispositif de mesures au col du lac Blanc. La station Dôme ne figure pas dans le champ de la photographie. AWS : Automatic Weather Station (station automatique de mesures météorologiques), SPC : Snow Particle Counter (compteur de particules de neige).

Le site du col du lac Blanc fait désormais partie du réseau Global Cryosphere Watch $(\mathrm{GCW})^{1}$ de l'Organisation météorologique mondiale (OMM).

Son référencement au sein du réseau Inarch (International Network for Alpine Research Catchment Hydrology) vient confirmer sa reconnaissance au sein de la communauté nationale et internationale. Le site constitue également une composante de CryobsClim $^{2}$ (la cryosphère, un observatoire du climat) labellisé en 2015 en tant que Système d'observation et d'expérimentation pour la recherche en environnement (Soere). Le Soere Cryobs-Clim regroupe sept laboratoires français et des partenaires étrangers avec une communauté de scientifiques qui s'intéressent au suivi des glaciers de montagne/calottes polaires et à l'étude de la neige et du permafrost de montagne. En 2017, le Soere Cryobs-Clim a rejoint l'Observatoire de la zone critique, applications, recherche (Ozcar).

Via le portail de Cryobs-Clim, les données du site du col du lac Blanc seront mises fin 2017 à disposition des scientifiques. Elles seront mises à jour à une fréquence annuelle à l'issue de chaque hiver après un contrôle qualité. En plus de cela, le Centre d'études de la neige a mis en œuvre un portail de visualisation en temps réel des données du site ouvert au public pendant la période allant de novembre à mai, avec accès aux images des webcams installées au site $^{3}$. Un accès virtuel via la plateforme de l'Irstea permet également de visualiser les observations acquises ${ }^{4}$.

\section{Un laboratoire d'étude du transport de neige par le vent}

Les différents observatoires constituant Cryobs-Clim ont une composante long terme, car la profondeur des observations doit permettre de déchiffrer les

1. http://globalcryospherewatch.org/cryonet/

2. https://cryobsclim.osug.fr

3. http://www.umr-cnrm.fr/cen/huez/PageWeb CLB.htm

4. http://monitoring-stations.grenoble.cemagref.fr/ Centrales_LB.php

Tableau 1. Mesures atmosphériques et de neige effectuées dans les stations du col du lac Blanc.

\begin{tabular}{|c|c|c|c|}
\hline \multicolumn{4}{|c|}{ Station Lac Blanc (Météo-France) } \\
\hline Variable & Début & Fin & Fréquence \\
\hline Épaisseur & 1989 & - & Horaire, infra-horaire \\
\hline Température & 1989 & - & Horaire, infra-horaire \\
\hline Humidité & 2011 & - & Horaire, infra-horaire \\
\hline Vent (vitesse et direction) & 1989 & - & Horaire, infra-horaire \\
\hline Rayonnement (montant) & 2011 & 2014 & Horaire, infra-horaire \\
\hline Rayonnement (descendant) & 2011 & 2014 & Horaire, infra-horaire \\
\hline \multicolumn{4}{|c|}{ Station Dôme (Météo-France) } \\
\hline Variable & Début & Fin & Fréquence \\
\hline Température & 2000 & - & Horaire, infra-horaire \\
\hline Vent (vitesse et direction) & 2000 & - & Horaire, infra-horaire \\
\hline \multicolumn{4}{|c|}{ Station La Muzelle (Météo-France) } \\
\hline Variable & Début & Fin & Fréquence \\
\hline Épaisseur & 2000 & - & Horaire, infra-horaire \\
\hline Rayonnement (montant) & 2011 & 2014 & Horaire, infra-horaire \\
\hline Rayonnement (descendant) & 2011 & 2014 & Horaire, infra-horaire \\
\hline \multicolumn{4}{|l|}{ Station Col (Irstea) } \\
\hline Variable & Début & Fin & Fréquence \\
\hline Épaisseur & 2008 & - & Horaire, infra-horaire \\
\hline Flux de neige transportée & 2008 & - & Horaire, infra-horaire \\
\hline Vent (vitesse et direction) & 2008 & - & Horaire, infra-horaire \\
\hline
\end{tabular}

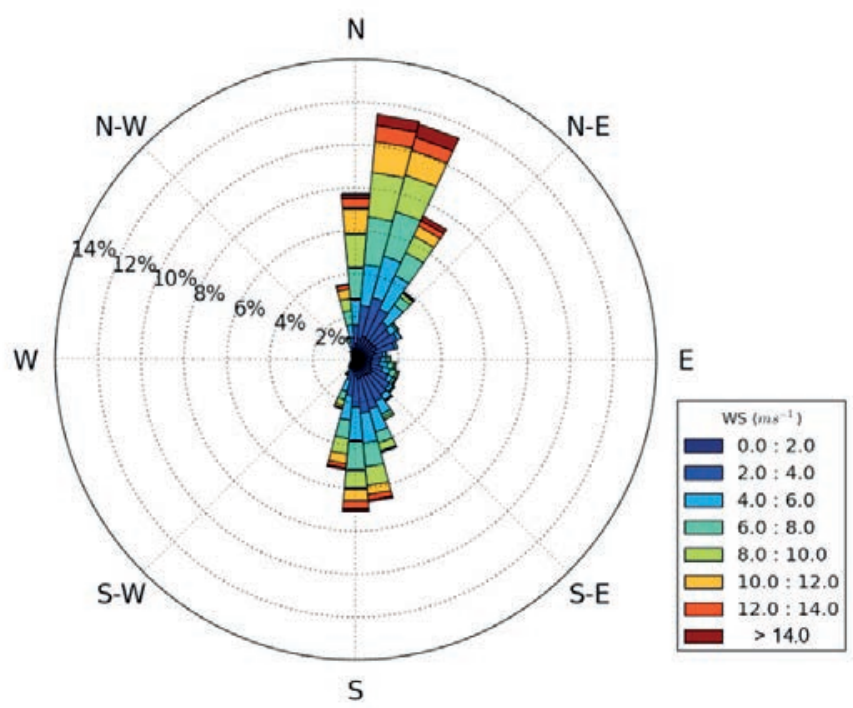

Figure 3. Rose des vents mesurés à la station Lac Blanc pour les hivers 2010/2011 à 2015/2016. 
changements sous pression naturelle ou anthropique. Les différents paramètres enregistrés au col du lac Blanc constituant cette composante «long terme » ont été décrits dans le tableau 1. Mais le col du lac Blanc est également un lieu d'expérimentation de plus court terme (campagnes de mesures intensives et de pointe limitées dans le temps) nécessaire à la compréhension de certains processus physiques pour des modélisations de plus en plus performantes. En effet, le transport de neige par le vent est un phénomène complexe représentatif des interactions entre l'atmosphère et le couvert neigeux, faisant intervenir plusieurs échelles spatio-temporelles. La connaissance fine de ces milieux est extrêmement difficile à acquérir avec des instruments de mesures qui restent encore à développer et un nombre de paramètres très élevé.

\section{Des moyens de mesures en constante évolution et développement}

Les mesures réalisées permettent certes d'améliorer la connaissance physique du phénomène, mais également de déterminer avec précision les entrées des modèles et/ou de valider lesdits modèles en confrontant sortie des modèles et mesures in situ (Naaim Bouvet et al., 2012).

Un des paramètres clés, dont la mesure est encore sujette à controverse, est le flux de neige transportée (NaaimBouvet, 2015). Au cours des vingt dernières années, ces mesures ont fait l'objet de nombreuses investigations au col du lac Blanc. Des capteurs mécaniques ont tout d'abord été utilisés : il s'agissait de «filets à papillon » exposés au vent capables de retenir la neige transportée tout en restant perméable au flux d'air. Ce dispositif nécessitait la présence d'expérimentateurs sur le site, ce qui limitait la fréquence d'échantillonnage et la durée des mesures. Depuis une collaboration avec le Japon, le site dispose depuis l'hiver 2009/2010 de trois Snow Particle Counter (SPC) visibles sur la figure 4. Ces capteurs, qui ont recours à un principe optique, détectent le diamètre et le nombre de particules de neige transportées par seconde. Des acquisitions à haute fréquence $(100 \mathrm{kHz})$ de signaux bruts couplés à des algorithmes spécifiques ont également permis de déterminer les vitesses de particules au sein de l'écoulement (Nishimura et al., 2014). Le capteur, de type industriel, sert également de capteur

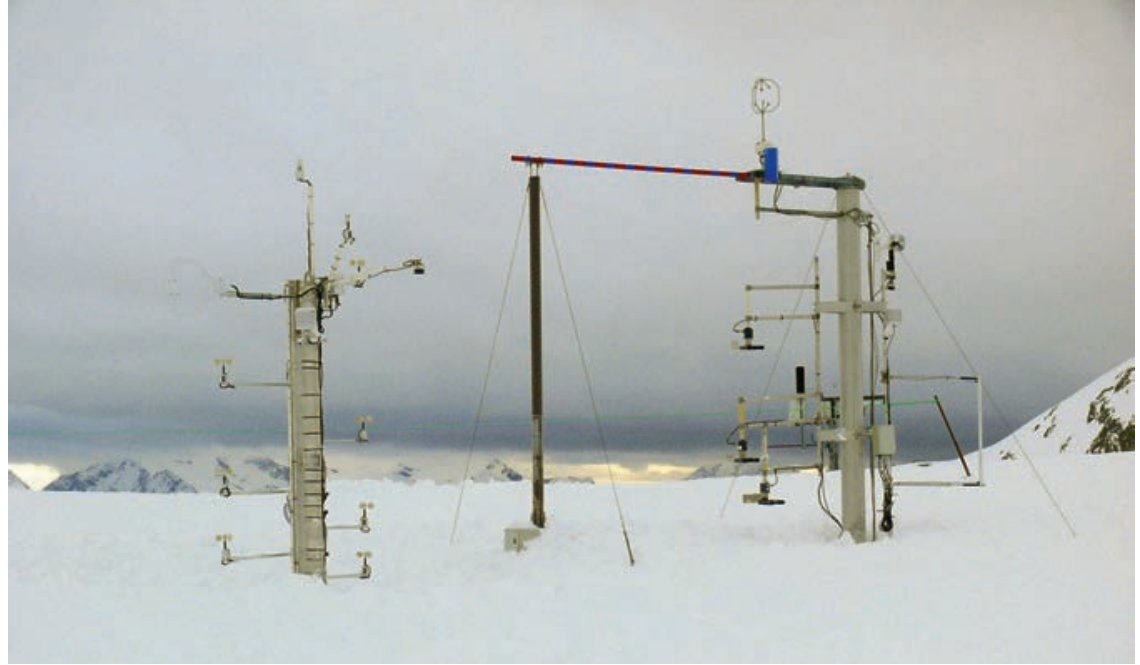

Figure 4. Banc de mesures de transport de neige par le vent. Crédit : H. Bellot, Irstea.

de référence sur un banc de mesures permettant de tester les différents capteurs disponibles sur le marché ou en cours de développement (Trouvilliez et al., 2015). En effet, un des enjeux est la spatialisation des observations, possible entre autres par l'implantation de capteurs à bas coût. D'une façon générale, du fait de son accessibilité et de la logistique associée, le site est très bien adapté pour tester de l'instrumentation en conditions extrêmes, avant une mission en Antarctique par exemple. Le site a donc fait l'objet de collaborations avec des industriels suisse et japonais et avec des laboratoires grenoblois (Laboratoire de glaciologie et de géophysique de l'environnement devenu en 2017 Institut des géosciences de l'environnement).

La répartition spatiale de la neige au sol et sa redistribution au cours des épisodes de transport constituent des paramètres de validation des modèles numériques. Dans les années 1990, un simple quadrillage par perches à neige avec relevé manuel hebdomadaire constituait le moyen d'estimer cette répartition spatiale. Les résolutions spatiale et temporelle étaient donc faibles. Dans les années 2000, un suivi photographique permettant l'estimation de l'albédo a permis d'identifier les zones de dépôt et d'érosion, augmentant ainsi la résolution spatiale (Corripio et al., 2004). Mais la véritable amélioration est venue de l'utilisation de mesures de télédétection. Un laser terrestre a ainsi été utilisé avec succès pour restituer la répartition spatiale des hauteurs de neige y compris sur les pentes raides (Richard, 2015 ; Schön et al., 2015). La stéréophotographie par drone a également été testée. Ces deux moyens de mesures sont utilisés de façon routinière au sein du Soere Cryobs-Clim.
La figure 5 montre l'évolution de la hauteur de neige autour du col du lac Blanc mesurée à l'aide du laser terrestre au cours de l'hiver 2015 (Richard, 2015). Ces cartes illustrent la variabilité spatiale très marquée de l'enneigement sur le site avec la formation de deux zones d'accumulation au nord et au sud du col. Ces zones sont caractérisées par des ruptures de pente marquées, au niveau desquelles la hauteur de neige peut dépasser localement $6 \mathrm{~m}$ au pic d'accumulation. Les distributions spatiales de hauteur de neige mesurées par laser terrestre et drone seront comparées à l'avenir aux mesures satellitaires à très haute résolution (Marti et al., 2016).

Si le problème de la résolution spatiale (la résolution inframétrique du laser terrestre est bien supérieure à ce qu'un modèle est capable de simuler aujourd'hui) est résolu, il n'en est rien de la résolution temporelle, car drone et laser terrestre nécessitent la présence d'un opérateur. Picard et al. (2015) ont donc développé un laser-scan automatique. Sa résolution spatiale est de $2 \mathrm{~cm}$ et il permet de couvrir une surface de $200 \mathrm{~m}^{2}$ en 4 heures. Ce système a été déployé au col du lac Blanc (NaaimBouvet et al., 2016). Il ne s'agit pas ici de valider un modèle numérique de transport (la surface scannée ne concerne que quelques mailles du modèle), mais d'étudier la formation des structures éoliennes (type sastrugi, ripples et dunes $^{5}$ ) visibles sur la figure 6 .

5. Formes éoliennes générées par le vent: (a) ripples et dunes sont formées par de la neige sans cohésion à l'image des formes observées dans les déserts de sable ; (b) sastrugi : formes d'érosion, dont l'existence est liée à la cohésion de la neige, aux arêtes tranchantes orientées dans la direction principale du vent. 

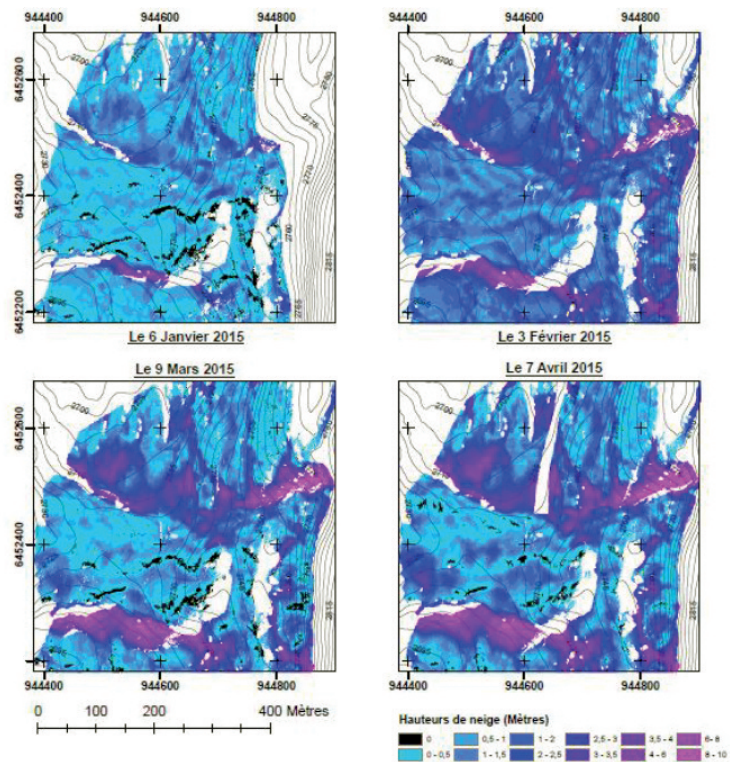

Figure 5. Cartes de hauteurs de neige (en mètres) à six dates au cours de la saison 2014-2015 obtenues par balayage laser terrestre. Cette série de cartes illustre le suivi de la formation de sastrugi par laser scan automatique.

Une autre expérience pilote en collaboration entre Isterre et le Centre d'études de la neige a été menée au col du lac Blanc afin de caractériser les conditions de surface de la neige en tirant parti des signaux GNSS (Global Navigation Satellite System) réfléchis autour d'une station géodésique de réception, installée à l'occasion de cette expérimentation, par la technique IPT (Interference Pattern Technique). La réflectométrie GNSS fournit ces informations de surface à une échelle intermédiaire par rapport aux observations in situ et satellitaires et permet de combler un manque d'observations en altitude. La méthode d'estimation de la hauteur de neige a été d'abord mise en œuvre en utilisant les sites existants du réseau français GNSS national permanent (RENAG). Lexpérimentation au col du lac Blanc a été menée afin de tester la méthode dans un environnement avec une topographie complexe, les estimations de hauteur de

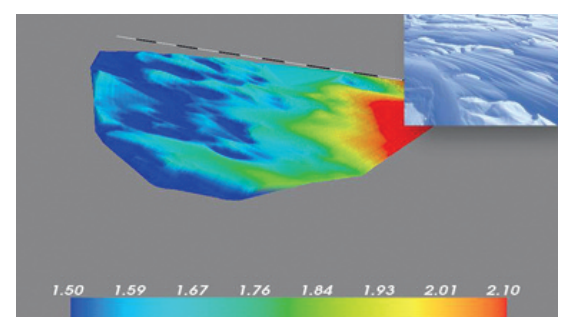

Figure 6. Suivi de formation de sastrugi par laser-scan automatique. La palette de couleurs indique la hauteur de neige (en mètres). La zone scannée couvre environ $70 \mathrm{~m}^{2}$. La photographie en incrustation représente un sastrugi similaire à ceux situés dans la zone de mesure.

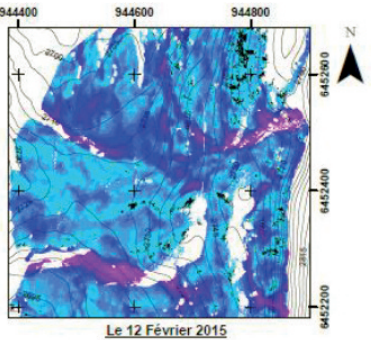

Le 12 Février 2015

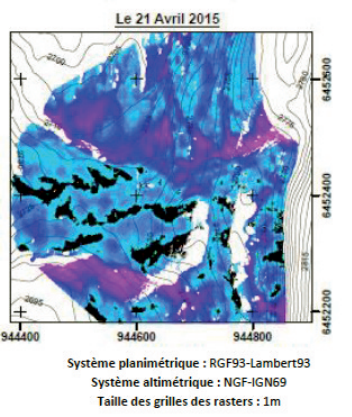

neige GNSS ont été évaluées à l'aide des mesures in situ et les simulations numériques sont issues du modèle de manteau neigeux Crocus (Boniface et al., 2015).

En matière d'instrumentation, la marge de progrès est encore grande. La mesure même des précipitations solides est notoirement difficile et ce d'autant plus en présence de vent (par exemple, Goodison et al., 1998). En cela, les territoires alpins sont semblables à l'Antarctique, où il est impossible d'estimer une climatologie des précipitations à partir d'observations in situ. Sans même parler d'estimation des précipitations solides, on a encore aujourd'hui des difficultés à identifier la présence d'une chute de neige pendant un épisode de transport (Naaim-Bouvet et al., 2014).

\section{Vers une meilleure compréhension des phénomènes physiques}

Pour ce qui est de l'étude des processus physiques, un des apports significatifs des données issues du col du lac Blanc a été la détermination de la vitesse seuil d'arrachement des différents types de neige (Guyomarc'h et Mérindol, 1998) qui est désormais utilisée dans les prévisions opérationnelles de transport de neige par le vent. En effet, la vitesse minimale nécessaire pour arracher une particule de neige peut varier de 4 à $10 \mathrm{~m} / \mathrm{s}$ ! La présence d'eau liquide au sein des couches de neige de surface empêche tout transport de neige du fait des effets de capillarité entre les grains. La figure 7 présente l'indice d'occurrence de transport fondée sur l'intensité du vent de surface et les caractéristiques des grains de neige au sol.

Les expérimentations menées ont également apporté de nouvelles données sur les flux de neige transportés, incluant : concentrations dans la couche de saltation (Naaim-Bouvet et al., 2010), vitesses des particules, distribution granulométrique des particules en loi gamma (figure 8), coefficient de diffusion et vitesses de chute des particules (Naaim-Bouvet et al., 2013), coefficient de rafales (Naaim-Bouvet et al., 2012), autant de paramètres qui sont des entrées des modèles numériques. La figure 8 illustre un exemple de distribution granulométrique

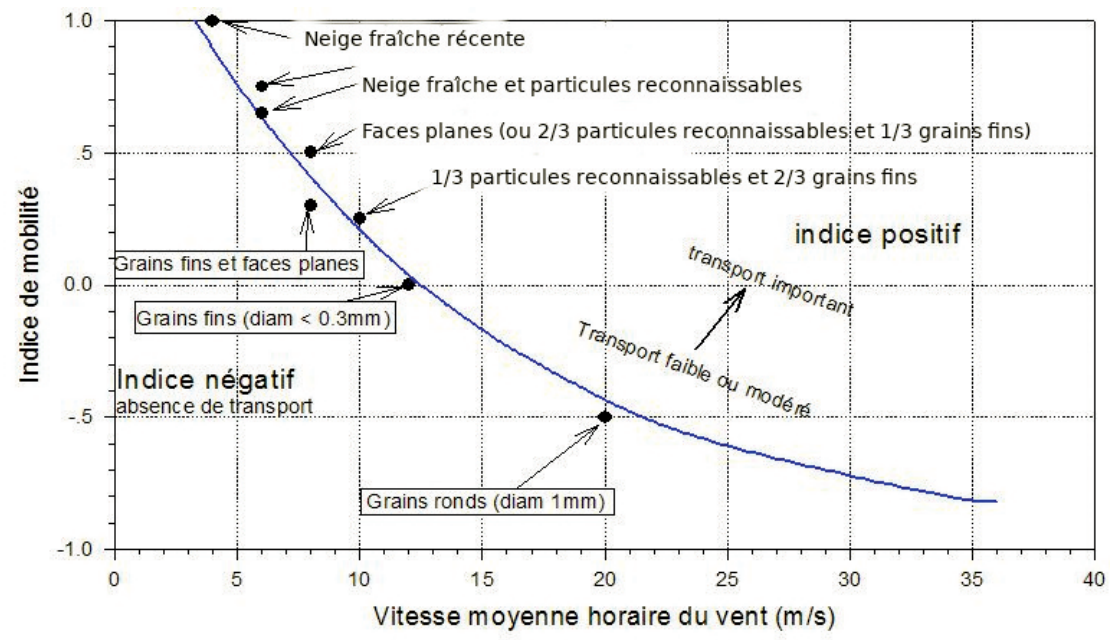

Figure 7. Indice d'occurrence de transport en fonction de la vitesse du vent (axe $x$ ) et de la mobilité potentielle des grains de surface du couvert neigeux (axe $y$ ). La mobilité est fonction des grains de neige en surface. 

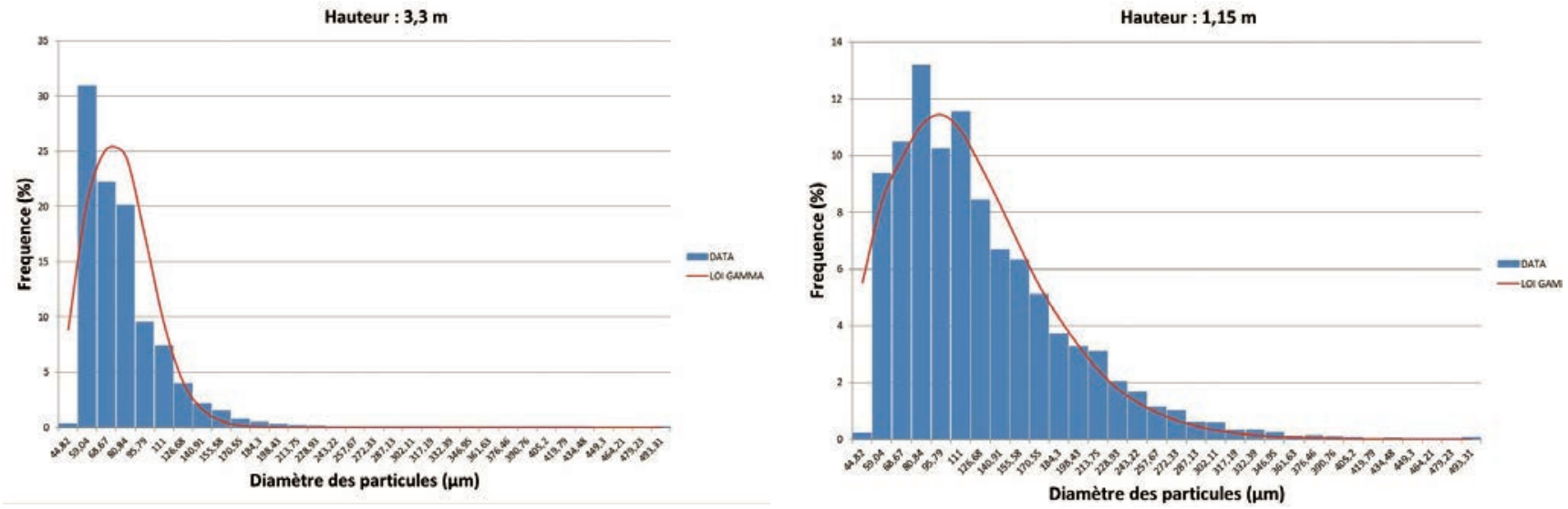

Figure 8. Distribution granulométrique des particules à une hauteur de 3,3 m (à gauche) et à une hauteur de 1,15 m (à droite).

des particules de neige transportées à deux hauteurs différentes. Ces mesures sont réalisées grâce au Snow Particles Counter (SPC). Dans la couche de diffusion turbulente, cette distribution suit une loi gamma. Le diamètre moyen des particules est d'autant plus faible que la hauteur au-dessus de la couche de neige est grande.

Si de nombreuses avancées ont pu être réalisées, il n'en reste pas moins que beaucoup reste encore à faire pour avoir accès à des données de plus en plus fines permettant de passer d'une vision macroscopique à une vision microscopique et de mieux identifier les effets de rétroaction. Ainsi, la prise en compte de la cohésion de la neige reste un défi, même si les vitesses seuil d'arrachement ont été déterminées en fonction des types de neige. En quoi la cohésion modifie-t-elle la prépondérance de l'entraînement par impact par rapport à l'entraînement aérodynamique et par la même la quantité de neige transportée dans la couche de saltation et la longueur nécessaire à l'obtention de la saturation ?
Quelle est la physique qui préside à la formation des formes éoliennes ? Quelle est leur rétroaction sur l'écoulement du vent et le flux de l'écoulement et par la même la quantité de neige transportée ? Des expérimentations conjointes en soufflerie climatique et in situ, que ce soit dans les Alpes ou en Antarctique, devrait apporter des éléments de réponse dans les années à venir. Le col du lac Blanc n'en a pas fini de jouer son rôle de Blizzard lab (Bolognesi et al., 2015).

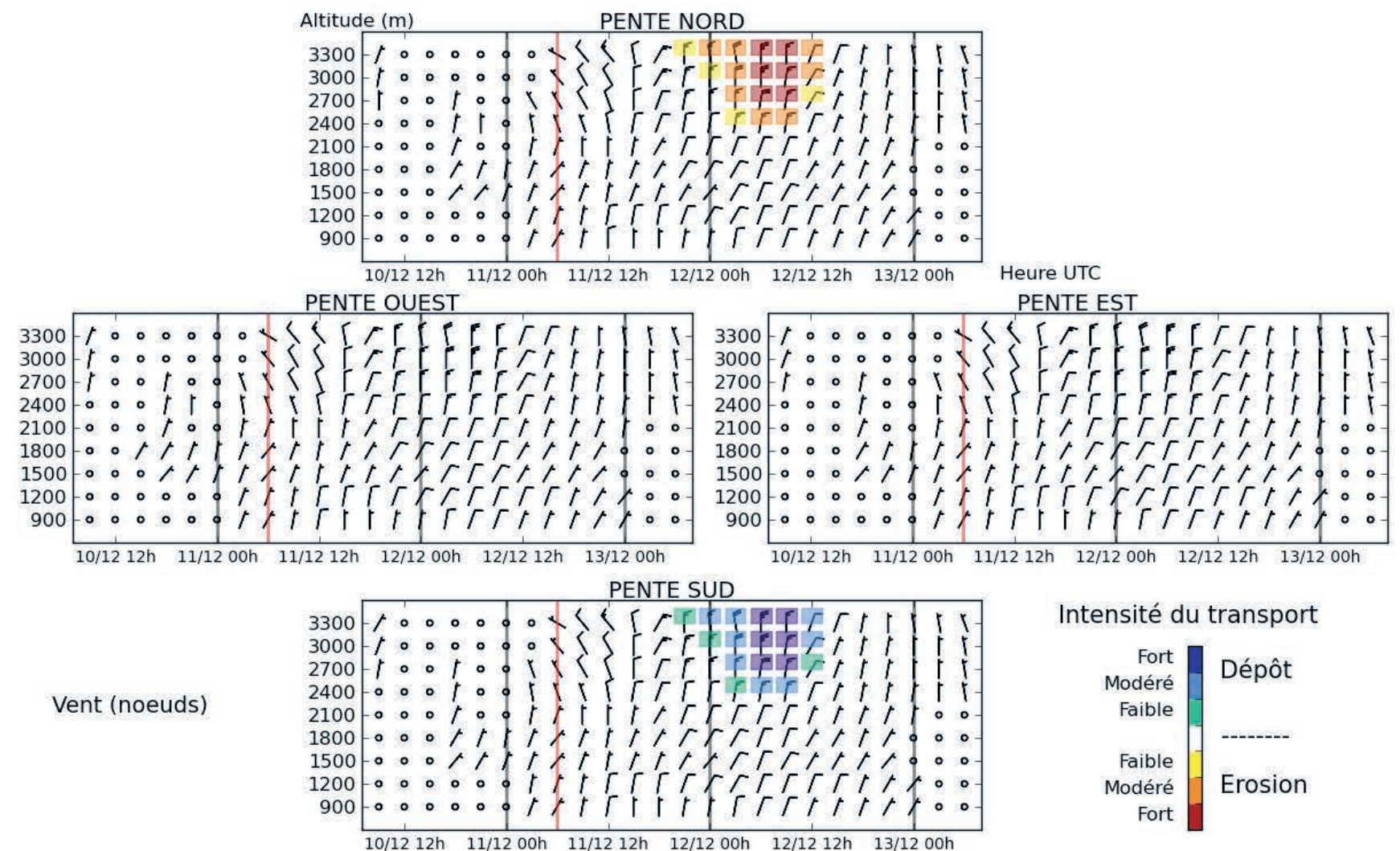

Figure 9. Prévisions de la chaîne de modélisation numérique S2M-Sytron par pas de temps de 3 h pour la période du 10 au 12 décembre 2016 et par pas d'altitude de $300 \mathrm{~m}$ pour le massif des Grandes Rousses. Les barbules indiquent la direction et l'intensité du vent. Les boîtes colorées indiquent l'intensité du transport de neige par le vent. Les prévisions sont disponibles pour quatre versants (nord, est, sud et ouest). 


\section{Prévision opérationnelle du transport de neige par le vent}

La détermination des vitesses seuil de transport en fonction des types de grain de neige en surface du manteau neigeux a contribué au développement du modèle de transport de neige par le vent Sytron (Durand et al., 2001). Sytron redistribue la neige selon des règles empiriques entre deux versants d'une crête virtuelle sur lesquels le manteau neigeux est simulé par le modèle détaillé Crocus (Brun et al., 1992 ; Vionnet et al., 2012). les couches de saltation et de suspension turbulente et inclut les pertes de masse par sublimation au cours du transport. Il tient également compte de la fragmentation des grains de neige lors du transport, facteur nécessaire pour bien appréhender l'évolution temporelle de la vitesse seuil de transport (Vionnet et al., 2013).

Sytron a été développé pour intégrer les effets du transport de neige par le vent dans la chaîne de prévision du risque d'avalanche utilisée à Météo-France. Il est ainsi utilisé de manière opérationnelle depuis l'hiver 2015/2016. Sytron fournit quotidiennement des analyses et des prévisions d'occurrence et d'intensité du transport de neige par le vent pour 23 massifs des Alpes françaises et 23 massifs des Pyrénées. Ces informations sont disponibles pour chaque massif par pas d'altitude de $300 \mathrm{~m}$ et pour huit orientations (nord, nord-est, est, sud-est, sud, sud-ouest, ouest, nord-ouest). Sytron simule le transport de neige dans

La figure 9 illustre un exemple de visualisation des prévisions de Sytron pour un épisode de transport de neige par le vent ayant eu lieu en décembre 2016 dans le massif des Grandes Rousses, dans lequel est situé le col du lac Blanc. Ce graphique permet aux prévisionnistes « avalanche » de pouvoir identifier dans un massif donné les altitudes et les expositions concernées par le transport de neige par le vent, ainsi que la chronologie prévue de l'épisode de transport. Vionnet et al. (2016) ont évalué en détail la version opérationnelle de Sytron en utilisant notamment les données du col du lac Blanc et ont montré les bonnes aptitudes du système à prévoir l'occurrence des épisodes de transport de neige par le vent (avec et sans chute de neige simultanée).

\section{Modélisation distribuée du transport de neige par le vent}

Le modèle Sytron repose sur une représentation idéalisée de la topographie. Afin de mieux représenter les effets locaux de redistribution de la neige par le vent, le col du lac Blanc a servi de site test pour le développement de modèles distribués de transport de neige par le vent. Ainsi, Durand et al. (2005) ont développé une version distribuée de Sytron qui a été utilisée pour simuler le transport de neige par le vent à une résolution horizontale de $45 \mathrm{~m}$ autour du col du lac Blanc. Ce modèle a été évalué en comparant la localisation des zones d'érosion et de dépôt simulées
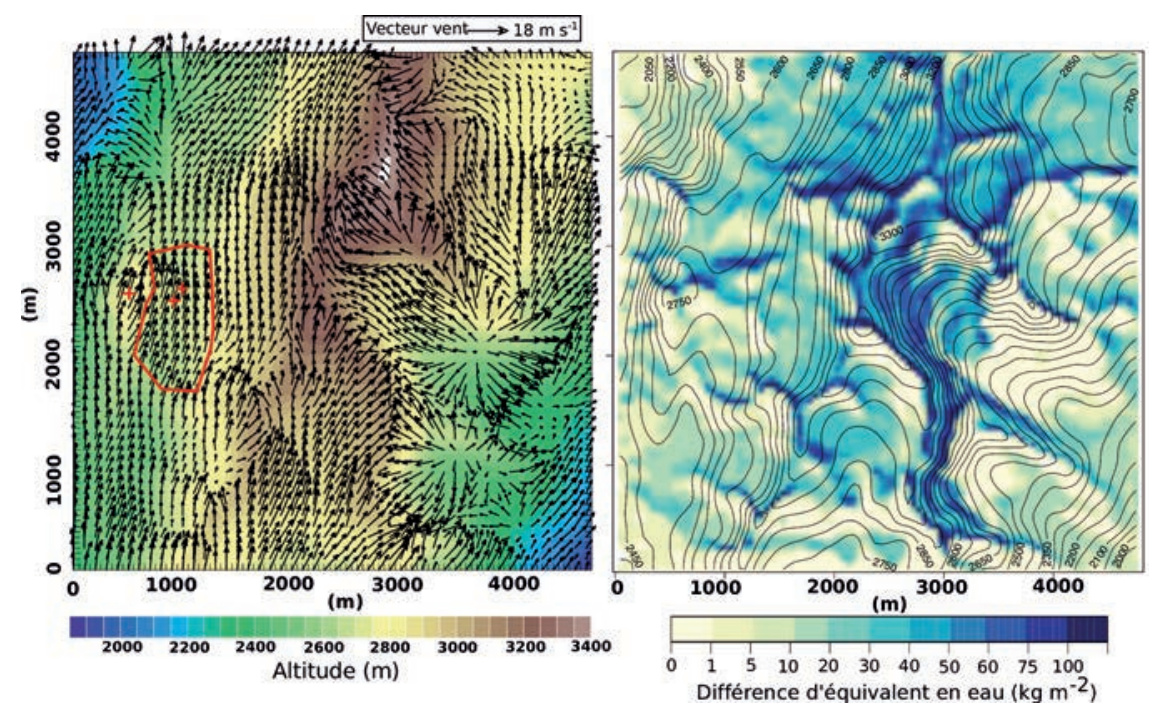

Figure 10. Gauche : champ de vent (flèches) à proximité de la surface (1,8-3 m) simulé par Meso-NH le 15/02/11 à 8 h 30. Les croix rouges désignent la localisation des stations automatiques et le contour rouge identifie la zone de canalisation du vent autour du col. Droite : différence d'équivalent en eau du manteau neigeux entre le 15/02/11 à 12 h et le 14/02/11 à 15 h. Les zones en bleu foncé indiquent les zones d'accumulation de la neige sous l'effet du vent. à celles observées sur le terrain par analyse photographique de la variabilité spatiale de l'albédo (Corripio et al., 2004). Cette version de Sytron était cependant limitée dans sa capacité à reproduire la complexité des processus à l'œuvre lors des épisodes de transport de neige par le vent (variabilité à petite échelle de précipitations, complexité du champ de vent, rétroactions de la sublimation sur la couche limite de surface...). Vionnet et al. (2014) ont donc proposé une alternative en développant le modèle couplé atmosphère/manteau neigeux Meso-NH/Crocus.

Dans cette approche, Meso-NH (Lafore et al., 1998) est utilisé jusqu'à une résolution horizontale de $50 \mathrm{~m}$ pour simuler l'évolution spatiale et temporelle des champs de vent et des précipitations. Il alimente le modèle Crocus qui simule l'évolution du manteau neigeux. En fonction de la vitesse du vent et des propriétés de la neige de surface, MesoNH/Crocus simule le transport de neige d'un point de grille à l'autre et intègre les effets de la sublimation liés au transport de neige par le vent. Meso$\mathrm{NH} / \mathrm{Crocus}$ a été évalué sur le site du col du lac Blanc en comparaison avec des données de flux et de diamètre des particules de neige issues du SPC, ainsi que des données de laser terrestre (Vionnet et al., 2014). Plus récemment, Guyomarch et al. (2016) ont utilisé le modèle Meso-NH/Crocus pour simuler la redistribution de la neige par le vent lors d'un épisode de chute de neige ventée de février 2011 autour du col du lac Blanc. La figure 10, tirée de ce travail, illustre les potentialités du modèle couplé surface/atmosphère. Sur la figure de gauche, le champ de vent à proximité de la surface (principalement de sud) est fortement influencé par la topographie. On remarque notamment des zones d'accélérations au niveau des crêtes et une canalisation de l'écoulement au niveau du col du lac Blanc (zone en rouge sur la figure) du fait de la topographie environnante. La figure de droite montre la carte d'accumulation de neige simulée avec de forts contrastes entre versant au vent et versant sous le vent.

Jusqu'à présent, Meso-NH/Crocus s'est avéré être un outil puissant pour la modélisation des interactions neige/atmosphère jusqu'à des échelles de l'ordre de 25-50 m en zone de montagne. En revanche, il ne peut pas représenter la variabilité spatiale à plus petite échelle, qui est pourtant marquée autour du col du lac Blanc comme en 
témoigne les cartes de hauteur de neige viseront à mettre en place des configurations du modèle à plus haute résolution en s'appuyant sur les développements en cours de schémas numériques optimisés et plus robustes. Par ailleurs, un effort portera sur la représentation de la variabilité spatiale du manteau neigeux à l'aide d'indices topographiques.

\section{Évaluation fine des performances des modèles météorologiques}

Depuis 2008, Météo-France s'appuie sur le modèle de prévision numérique du temps Arome pour réaliser les prévisions à courte échéance (Seity et al., 2011 ; Brousseau et al., 2016). Avec sa résolution horizontale kilométrique (2,5 km jusqu'en 2015, 1,3 km depuis), Arome propose une représentation relativement détaillée de la topographie en zone de montagne et présente un fort neigeux en zone de montagne (Quéno et al., 2016 ; Vionnet et al., 2016). Depuis 2016, le site du col du lac Blanc est utilisé pour le suivi des performances du modèle Arome à haute altitude. La figure 11 montre l'exemple de l'évaluation de la vitesse du vent (moyenne et maximale) simulée par Blanc. Afin de compléter cette évaluation, un dispositif avancé a été mis en place lors de l'été 2017 et servira dès l'hiver 2017/2018. Ce dispositif se compose d'un profil vertical de température et d'humidité jusqu'à $6 \mathrm{~m}$ de la figure 5. De futurs développements potentiel pour la simulation du manteau Arome et observée à la station Lac

au-dessus de la surface du manteau neigeux et d'un ensemble de radiomètres pour calculer le bilan radiatif net à la surface de la neige.

\section{Conclusions}

Les mesures de transport de neige en montagne, et les mesures nivométéorologiques en haute montagne en général, sont assez rares, ce qui fait du site du col du lac Blanc un observatoire unique. Il a ainsi été récemment reconnu au sein de la communauté nationale et internationale via différentes labellisations. Une de nos premières ambitions pour ce site est qu'il demeure un site de référence fiable en observations de haute montagne. Sa panoplie de mesures sera complétée en 2018 avec l'installation pérenne de nouveaux capteurs de mesures du rayonnement descendant et montant. Jusqu'ici, les mesures de rayonnement effectuées au site étaient plutôt ponctuelles.

Ce dispositif de mesures en continu garantit la pérennité des travaux de recherches sur la compréhension et la modélisation de l'effet du transport de neige par le vent. Ce dispositif permet également d'explorer de nouvelles pistes pour une meilleure prise en compte de la variabilité spatiale extrêmement complexe du milieu montagne pour des prévisions du risque d'avalanche à des échelles de temps et d'espace plus pertinentes qu'actuellement. Les évaluations systématiques des champs d'analyses et de prévisions numériques à haute
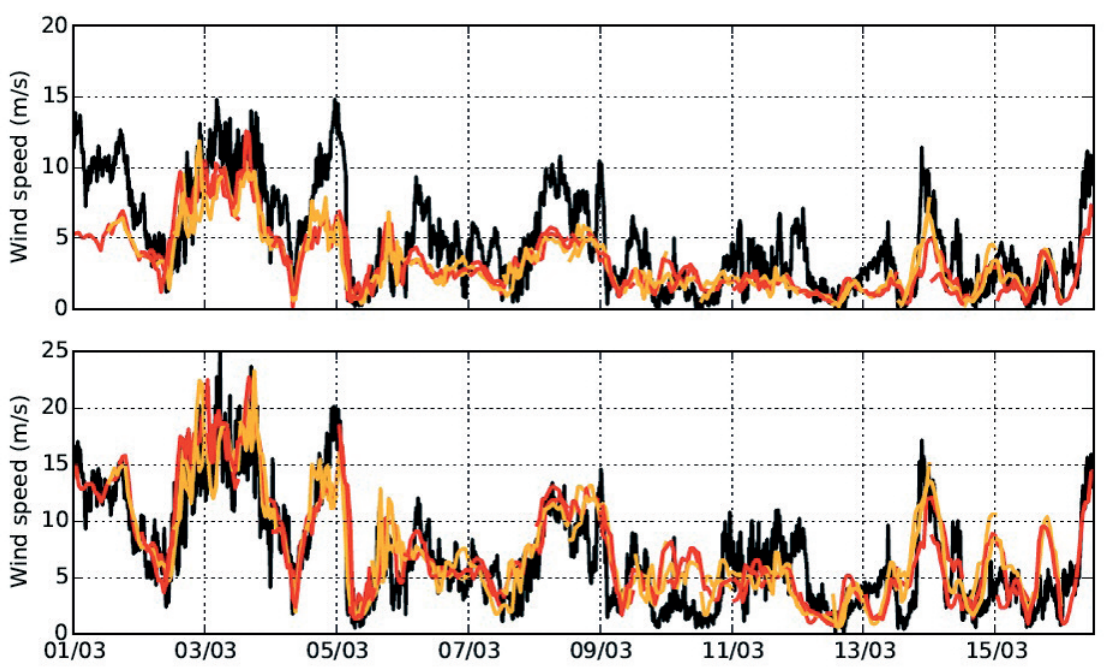

Figure 11. Vitesse moyenne (haut) et maximale (bas) du vent au col du lac Blanc : Observations (noir) et prévisions du modèle Arome (réseau de 00 h 00 UTC : rouge; réseau de 12 h 00 UTC : orange) pour la période du $1^{\mathrm{er}}$ au 16 mars 2016. résolution au col du lac Blanc permettront d'envisager de nouvelles utilisations du modèle Arome dans ses formes déterministe et ensembliste, comme le potentiel des rafales de vent du modèle pour cartographier le transport de neige. Plus généralement, l'instrumentation du site sera au cœur des développements visant à une meilleure prise en compte des effets du transport de neige par le vent (redistribution, effet du la neige de surface...) dans les futures chaînes de modélisation du manteau neigeux et du risque d'avalanches.

En ce qui concerne la télédétection spatiale, le site du col du lac Blanc est de plus en plus prisé pour différentes études visant à mieux suivre l'évolution du manteau neigeux, et du risque qui découle de son instabilité, en exploitant les mesures de télédétection haute et très haute résolution. Une part importante de ces recherches est réalisée dans le cadre du projet Cnes Kalideos-Alpes piloté par le Centre d'études de la neige (https://alpes.kalideos.fr). Le groupement Kalideos-Alpes, constitué de plusieurs laboratoires et institutions de recherche, ambitionne de favoriser l'usage des observations de télédétection et le développement de méthodes novatrices dans des thématiques aussi variées que le suivi de la végétation, de la neige, de l'évolution des glaciers et des risques gravitaires. Les images satellitaires cibles du groupement Kalideos-Alpes sont les images optiques de toutes sortes incluant les images récentes de Pleiades $(50 \mathrm{~cm})$, Spot-6 $(1,5 \mathrm{~m})$, Spot-7 (1,5 m), Sentinel-2 (10 m, $20 \mathrm{~m}, 60 \mathrm{~m})$, les images SAR de Sentinel-1 (20 m), Alos-2 (3 m, $6 \mathrm{~m}$, $10 \mathrm{~m})$, Terrasar- $X(25 \mathrm{~cm}, 3 \mathrm{~m}$, $19 \mathrm{~m})$. En plus des programmations d'acquisitions, une archive satellitaire est en cours de construction pour un accès à plus de 20 ans de données.

La zone Kalideos-Alpes a été choisie pour inclure différents sites d'intérêt dont le site du col du lac Blanc. Plusieurs études sont en cours ou prévues au site et notamment les travaux d'intercomparaisons des mesures actives micro-ondes issues de Sentinel-1, Alos-2 et Terrasar-X pour la caractérisation de certaines propriétés de la neige, l'apport des mesures satellitaires pour cartographier les dépôts d'avalanches et le suivi des estimations de hauteurs de neige par imagerie optique haute résolution (Pleiades notamment). 
Une des grandes ambitions du site est de devenir un site pionnier dans l'expérimentation instrumentale en haute montagne : le site offre de nombreux avantages le rendant attractif pour tester de nouvelles approches ou expérimentations. Cela fut déjà le cas avec des expérimentations menées en partenariat avec différents laboratoires de recherche afin de tester une approche expérimentale et/ou de modélisation.

\section{Remerciements}

Les auteurs remercient la Société d'aménagement touristique de l'Alpe d'Huez pour son soutien logistique constant ainsi que la Région RhôneAlpes, le Pôle alpin d'études et de recherche pour la prévention des risques naturels, l'Observatoire des sciences de l'Univers de Grenoble (Osug), l'Institut des sciences de l'Univers du CNRS, et les programmes Interreg (Dynaval et MAP3) pour leur soutien financier. Ils remercient également leurs collègues Robert Bolognesi, Karen Boniface, Thierry Castelle, Javier Corripio, François-Xavier Cierco, Laurent Merindol, Kiouchi Nishimura, Jean- Luc Michaux, Frédéric Ousset, Jean-Michel Panel, Alexander Prokop, Philippe Puglièse, Aymeric Richard, Firmin Fontaine Xavier Ravanat et Martine Roussel, pour leurs participations actives aux expérimentations menées au col du lac Blanc depuis 20 ans.

\section{Bibliographie}

Aksamit N.O., Pomeroy J.W., 2016. Near-surface snow particle dynamics from particle tracking velocimetry and turbulence measurements during alpine blowing snow storms. The Cryosphere, 10, 3043-3062.

Bolognesi R., Guyomarc'h G., Naaim-Bouvet F., 2015. Blizzard Lab, Météo Magazine, 14, 26-29

Boniface K., Walpersdorf A., Guyomarc'h G., Deliot Y., Karbou F., Vionnet V., Nievinski F., 2015. GNSS reflectometry measurement of snow depth and soil moisture in the French Alps. International Geoscience and Remote Sensing Symposium, 5205-5207, 26-31 July 2015. doi: 10.1109/IGARSS.2015.7327007

Brousseau P., Seity Y., Ricard D., Léger J., 2016. Improvement of the forecast of convective activity from the AROME-France system. Q. J. R. Meteorol. Soc., 142, 2231 -2243.

Brun E., David P., Sudul M., Brunot G., 1992. A numerical model to simulate snow-cover stratigraphy for operational avalanche forecasting. J. Glaciol., 38 , $13-22$.

Comola F., Kok J.F., Gaume J., Paterna E., Lehning M., 2017. Fragmentation of wind-blown snow crystals. Geophys. Res. Lett., 44, 4195-4203.

Corripio J.G., Durand Y., Guyomarc'h G., Mérindol L., Lecorps D., Pugliese P., 2004. Land-based remote sensing of snow for the validation of a snow transport model. Cold Reg. Sci. Technol., 39, 93-104.

Durand Y., Guyomarc'h G., Mérindol L., 2001. Numerical experiments of wind transport over a mountainous instrumented site: I. Regional scale. Ann. Glaciol., 32, 187-194.

Durand Y., Guyomarc'h G., Mérindol L., Corripio J.G., 2005. Improvement of a numerical snow drift model and field validation. Cold Reg. Sci. Technol., 43, 93-103.

Goodison B.E., Louie P.Y.T., Yang D., 1998. Solid precipitation measurement intercomparison, WMO/TD - No. 872, WM0, Instrument and Observing Methods report No. 67, WMO, Geneva.

Guyomarc'h G., Mérindol L., 1998. Validation of an application for forecasting blowing snow. Ann. Glaciol., 26, 138-14.

Guyomarch G., Vionnet V., Lafaysse M., Giraud G., Déliot Y., Naaim-Bouvet F., 2016. description and evaluation of the new french operational chain of models for avalanche hazard forecasting incorporating a drifting snow scheme. The International Snow Science Workshop 2016. proceeding available from http://arc.lib.montana.edu/snowscience/item.php?id=2447.

Groot Zwaaftink, C.D., Löwe H., Mott R., Bavay M., Lehning M., 2011. Drifting snow sublimation: A high-resolution 3-D model with temperature and moisture feedbacks. J. Geophys. Res. Atmos., 116, D16107. doi: 10.1029/2011JD015754.

Lafore J.-P., Stein J., Asencio N., Bougeault P., Ducrocq V., Duron J., Pinty J.-P., 1997. The Meso-NH atmospheric simulation system. Part I: Adiabatic formulation and control simulations. Ann. Geophys., 16, 90-109.

Lehning M., Löwe H., Ryser M., Raderschall N., 2008. Inhomogeneous precipitation distribution and snow transport in steep terrain. Water Resour. Res., 44, W07404. doi: 10.1029/2007WR006545.

Liston G.E., Haehnel R.B., Sturm M., Hiemstra C.A., Berezovskaya S., Tabler R.D., 2007. Instruments and methods simulating complex snow distributions in windy environments using SnowTran-3D. J. Glaciol., 53, 241-256.

MacDonald M.K., Pomeroy J.W., Pietroniro A., 2010. On the importance of sublimation to an alpine snow mass balance in the Canadian Rocky Mountains. Hydrol. Earth Syst. Sci., 14, 1401-1415

Marti R., Gascoin S., Berthier E., de Pinel M., Houet T., Laffly D., 2016. Mapping snow depth in open alpine terrain from stereo satellite imagery. The Cryosphere, $10,1361-1380$. doi: 10.5194/tc-10-1361-2016

Naaim M., Naaim-Bouvet F., Martinez H., 1998. Numerical simulation of drifting snow: erosion and deposition models. Ann. Glaciol., 26, 191-196.

Naaim-Bouvet F., Bellot H., Naaim M., 2010. Back analysis of drifting-snow measurements over an instrumented mountainous site. Ann. Glaciol., 51, 207-217.

Naaim-Bouvet F., Naaim M., Bellot H., Nishimura K., 2011. Wind and drifting snow gust factor in an Alpine context. Ann. Glaciol., 58, 223-230.

Naaim-Bouvet F., Guyomarc'h G., Naaim M., Durand Y., Bellot H., Pugliese P., 2012. Transport de neige par le vent sur un site de haute-montagne : de la modélisation à I'observation, de l'observation à la modélisation. La Houille Blanche, 1, 51-57.

Naaim-Bouvet F., Bellot H., Naaim M., 2013. Size distribution, Schmidt number and terminal velocity of blowing snow particles. International Snow Science Workshop, Grenoble, Chamonix Mont-Blanc, 140-146.

Naaim-Bouvet F., Bellot H., Nishimura K., Genthon C., Palerme C., Guyomarc'h G., Vionnet V., 2014. Detection of snowfall occurrence during blowing snow events using photoelectric sensors. Cold Reg. Sci. Technol. 106-107, 11-21. doi: 10.1016/j.coldregions.2014.05.005

Naaim-Bouvet F., 2015. Des capteurs dans le blizzard. Météo Magazine, 14, 31-35.

Naaim-Bouvet F., Picard G., Bellot H., Arnaud L., Vionnet V., 2016. Snow surface roughness monitoring using time-lapse terrestrial laserscan. International Snow Science Workshop. Proceeding available from http://arc.lib.montana.edu/snow-science/item.php?id=2340

Nishimura K., Yokoyama C., Ito Y., Nemoto M., Naaim-Bouvet F., Bellot H., Fujita K., 2014. Snow particle speeds in drifting snow. J. Geophys. Res. Atmos., 119, 9901-9913. doi: 10.1002/2014JD021686 
Picard G., Arnaud L., Panel J.-M., Morin S., 2016. Design of a scanning laser meter for monitoring the spatio-temporal evolution of snow depth and its application in the Alps and in Antarctica. The Cryosphere, 10, 1495-1511.

Quéno L., Vionnet V., Dombrowski-Etchevers I., Lafaysse M., Dumont M., Karbou F., 2016. Snowpack modelling in the Pyrenees driven by kilometric-resolution meteorological forecasts. The Cryosphere, 10, 1571-1589.

Richard A., 2015. Suivi de l'enneigement saisonnier sur un site de haute montagne par balayage laser terrestre. Mémoire de stage de fin d'études, Insa Strasbourg

Schon P., Prokop A., Vionnet V., Guyomarc'h G., Naaim-Bouvet F., Heiser M., 2015. Improving a terrain-based parameter for the assessment of snow depths with TLS data in the Col du Lac Blanc area. Cold Reg. Sci. Technol., 114, 15-26. doi: 10.1016/j.coldregions.2015.02.005

Schirmer M., Wirz V., Clifton A., Lehning M., 2011. Persistence in intra-annual snow depth distribution: 1. Measurements and topographic control. Water Resour. Res., 47, W09516. doi: 10.1029/2010WR009426.

Schmidt R.A., 1982. Vertical profiles of wind speed, snow concentration, and humidity in blowing snow. Boundary-Layer Meteorol., 23, 223-246.

Schneiderbauer S., Prokop A., 2011. The atmospheric snow-transport model: SnowDrift3D. J. Glaciol., 57, 526-542.

Schweizer J., Bruce Jamieson J., Schneebeli M., 2003. Snow avalanche formation. Rev. Geophys., 41, 1016. doi: 10.1029/2002RG000123, 2003.

Seity Y., Brousseau P., Malardel S., Hello G., Bénard P., Bouttier F., Masson V., 2011. The AROME-France convective-scale operational model. Mon. Weather Rev., 139, $976-991$.

Trouvilliez A., Naaim-Bouvet F., Bellot H., Genthon C., Gallée H., 2015. Evaluation of the flowcapt acoustic sensor for the aeolian transport of snow. J. Atmos. Oceanic Technol., 32, 1630-1641.

Vionnet V., Brun E., Morin S., Boone A., Faroux S., Le Moigne P., Willemet J.M., 2012. The detailed snowpack scheme Crocus and its implementation in SURFEX v7.2. Geosci.ic Model Dev., 5, 773-791.

Vionnet V., Guyomarc'h G., Naaim-Bouvet F., Martin E., Durand Y., Bel C., Bellot H., Puglièse P., 2013. Occurrence of blowing snow events at an alpine site over a 10-year period: observations and modeling. Adv. Water Res., 55, 53-63.

Vionnet V., Dombrowski-Etchevers I., Lafaysse M., Quéno L., Seity Y., Bazile E., 2016. Numerical weather forecasts at kilometer scale in the French Alps: Evaluation and application for snowpack modeling. J. Hydrometeorol., 17, 2591-2614.

Vionnet V., Martin E., Masson V., Lac C., Bouvet F.N., Guyomarc'h G. 2017. High resolution large eddy simulation of snow accumulation in alpine terrain. J. Geophys. Res. Atmos., accepted for publication. doi: 10.1002/2017JD026947

Winstral A., Marks D., Gurney R., 2013. Simulating wind-affected snow accumulations at catchment to basin scales. Adv. Water Res., 55, 64-79. 